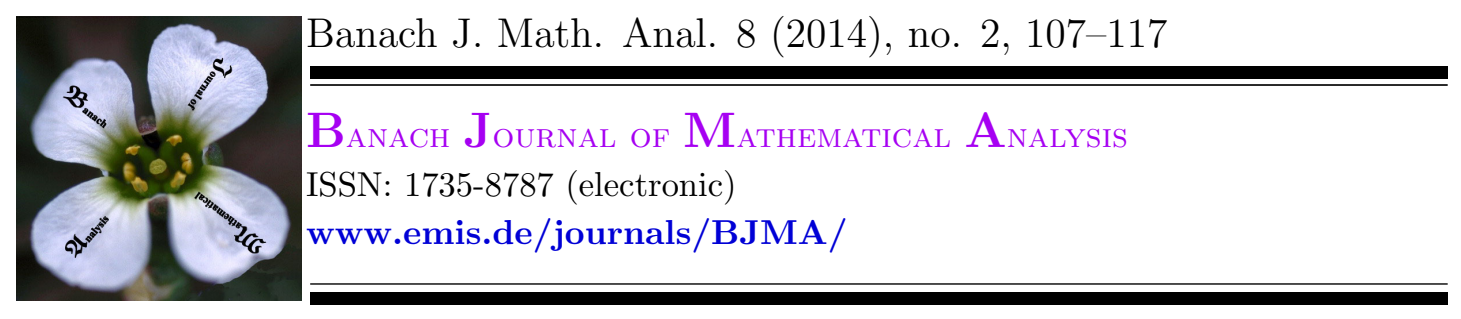

\title{
GENERALIZED BIPROJECTIVITY AND BIFLATNESS OF ABSTRACT SEGAL ALGEBRAS
}

\author{
FATEMEH ABTAHI
}

Communicated by Y. Zhang

\begin{abstract}
We investigate generalized amenability, contractibility, biprojectivity and biflatness properties of various classes of abstract Segal algebras with respect to the Banach algebra $\mathcal{A}$. Moreover, we verify some of the previous available results about Segal algebras, for abstract Segal algebras.
\end{abstract}

\section{INTRODUCTION AND PRELIMINARIES}

Let $\mathcal{A}$ be a Banach algebra. Following Johnson [13], we say that $\mathcal{A}$ is amenable if it has a bounded approximate diagonal, i.e., there is a bounded net $\left(m_{\lambda}\right)$ in the projective tensor product $\mathcal{A} \widehat{\otimes} \mathcal{A}$ such that $\left\|a m_{\lambda}-m_{\lambda} a\right\|_{\mathcal{A} \widehat{\otimes} \mathcal{A}} \rightarrow_{\lambda} 0$ and $\left\|a \pi_{\mathcal{A}}\left(m_{\lambda}\right)-a\right\|_{\mathcal{A}} \rightarrow_{\lambda} 0$, for each $a \in \mathcal{A}$. Here and in the sequel, $\pi_{\mathcal{A}}$ always denotes the product morphism from $\mathcal{A} \widehat{\otimes} \mathcal{A}$ into $\mathcal{A}$, specified by $\pi_{\mathcal{A}}(a \otimes b)=a b$. Similarly, $\mathcal{A}$ is contractible if and only if it has a diagonal, i.e., there is an element $m \in \mathcal{A} \widehat{\otimes} \mathcal{A}$ for which $a m=m a$ and $\pi_{\mathcal{A}}(m) a=a$, for all $a \in \mathcal{A}$ [8]. In [7], it was introduced and studied a notion of amenability based on existence of an approximate diagonal (not necessarily bounded). In fact $\mathcal{A}$ is called pseudoamenable if there is a net $\left(m_{\lambda}\right) \subseteq \mathcal{A} \widehat{\otimes} \mathcal{A}$, called an approximate diagonal for $\mathcal{A}$, such that $\left\|a m_{\lambda}-m_{\lambda} a\right\|_{\mathcal{A} \widehat{\otimes} \mathcal{A}} \rightarrow_{\lambda} 0$ and $\left\|a \pi_{\mathcal{A}}\left(m_{\lambda}\right)-a\right\|_{\mathcal{A}} \rightarrow_{\lambda} 0$, for each $a \in \mathcal{A}$. Moreover, $\mathcal{A}$ is called pseudo-contractible if it has a central approximate diagonal, i.e., an approximate diagonal $\left(m_{\lambda}\right)$ satisfying $a m_{\lambda}=m_{\lambda} a$ for all $a \in \mathcal{A}$ and all $m_{\lambda}$

Date: Received: Apr. 1, 2013; Revised: Aug. 31, 2013; Accepted: Oct. 5, 2013.

2010 Mathematics Subject Classification. Primary 46H25; Secondary 43A07.

Key words and phrases. (pseudo- $\phi-)$ amenability, (approximate) biflatness, (approximate) biprojectivity, $\phi$-contractibility, pseudo-contractibility. 
Let $\phi \in \sigma(\mathcal{A})$, consisting of all nonzero characters on $\mathcal{A}$. The concept of $\phi$-amenability for Banach algebras was introduced by Kaniuth et al. [14, 15]. It is in fact a generalization of the left amenability of Lau algebras. Precisely, $\mathcal{A}$ is called $\phi$-amenable if there exists a bounded net $\left(a_{\alpha}\right) \in \mathcal{A}$ such that

$$
\phi\left(a_{\alpha}\right) \rightarrow_{\alpha} 1 \text { and }\left\|a a_{\alpha}-\phi(a) a_{\alpha}\right\|_{\mathcal{A}} \rightarrow_{\alpha} 0,
$$

for each $a \in \mathcal{A}$. Moreover, the notion of $\phi$-contractibility of $\mathcal{A}$ was introduced and studied by $\mathrm{Hu}$ et al. [11]. In fact, $\mathcal{A}$ is called $\phi$-contractible if there exists a $\phi$-diagonal for $\mathcal{A}$; that is, an element $m \in \mathcal{A} \widehat{\otimes} \mathcal{A}$ such that

$$
\phi\left(\pi_{\mathcal{A}}(m)\right)=1 \quad \text { and } \quad a . m=\phi(a) m,
$$

for each $a \in \mathcal{A}$. Also in [3], it was introduced a new definition of amenability which was related to homomorphisms of Banach algebras, and then weak amenability of Banach algebras was generalized.

For completeness, we also recall the definitions and basic relationships of the standard homological properties. We refer to [9], as a standard reference in this field. Following this reference, we say that $\mathcal{A}$ is biprojective if there is a bounded $\mathcal{A}$-bimodule map $\xi: \mathcal{A} \rightarrow \mathcal{A} \widehat{\otimes} \mathcal{A}$ such that $\pi_{\mathcal{A}} \circ \xi=i d_{\mathcal{A}}$. Also $\mathcal{A}$ is biflat if there is a bounded $\mathcal{A}$-bimodule map $\theta:(\mathcal{A} \widehat{\otimes} \mathcal{A})^{*} \rightarrow \mathcal{A}^{*}$ such that $\theta \circ \pi_{\mathcal{A}}{ }^{*}=i d_{\mathcal{A}^{*}}$. We also remind from [20] that $\mathcal{A}$ is approximately biprojective if there is a net $\left(\xi_{\lambda}\right)$ of bounded $\mathcal{A}$-bimodule morphisms from $\mathcal{A}$ into $\mathcal{A} \widehat{\otimes} \mathcal{A}$ such that

$$
\left\|\pi_{\mathcal{A}} \circ \xi_{\lambda}(a)-a\right\|_{\mathcal{A}} \rightarrow_{\lambda} 0,
$$

for each $a \in \mathcal{A}$. Furthermore, $\mathcal{A}$ is called approximately biflat if there is a net $\theta_{\delta}:(\mathcal{A} \widehat{\otimes} \mathcal{A})^{*} \rightarrow \mathcal{A}^{*},(\delta \in \Delta)$, of bounded $\mathcal{A}$-bimodule morphisms such that $W^{*} O T-\lim _{\delta} \theta_{\delta} \circ \pi_{\mathcal{A}}{ }^{*}=i d_{\mathcal{A}^{*}}$, where $\left(W^{*} O T\right)$ is the weak ${ }^{*}$ operator topology on $B\left(\mathcal{A}^{*}\right)$ [19]. Recall that the weak* operator topology $\left(W^{*} O T\right)$ on $B\left(\mathcal{A}^{*}\right)$ is the locally convex topology determined by the seminorms $\left\{p_{a, g}: a \in \mathcal{A}, g \in \mathcal{A}^{*}\right\}$, where $p_{a, g}(T)=|\langle g, T(a)\rangle|$, for all $a \in \mathcal{A}$ and $g \in \mathcal{A}^{*}$.

Let $\mathcal{B}$ be an abstract Segal algebra with respect to $\mathcal{A}$. It was characterized character amenability and character contractibility of abstract Segal algebras. Specially it was shown that $\phi$-amenability ( $\phi$-contractibility) of $\mathcal{A}$ is equivalent to $\left.\phi\right|_{\mathcal{B}}$-amenability $\left(\left.\phi\right|_{\mathcal{B}}\right.$-contractibility) of $\mathcal{B}$, where $\phi \in \sigma(\mathcal{A})$; see [1].

The main motivations for this work, stem from [19], which gives several results on the generalized notions of amenability and biflatness of Segal algebras associated with locally compact groups. In the first section of the present paper, we introduce the concept of pseudo- $\phi$-amenability of the Banach algebra $\mathcal{A}$. Then we show that pseudo-amenability of $\mathcal{A}$ implies pseudo- $\phi$-amenability of $\mathcal{A}$, for each $\phi \in \sigma(\mathcal{A})$. Also we prove that if $\mathcal{B}$ is pseudo-amenable then $\mathcal{A}$ is pseudo- $\phi$-amenable. Moreover, we introduce some sufficient conditions to get an equivalency between amenability of $\mathcal{A}$ and pseudo-amenability of $\mathcal{B}$. We also investigate the previous results for the concept of contractibility and also pseudo-contractibility. The last section is devoted to approximate biprojectivity and biflatness of abstract Segal algebras. The main results of the present work are given in this section. In fact we make some attempts to investigate some of the aforementioned arguments for abstract Segal algebras. For example, we 
show that in some circumstances, biprojectivity $\mathcal{A}$ (resp. $\mathcal{B}$ ) implies approximate biprojectivity of $B$ (resp. $\mathcal{A}$ ). Mainly, as the converse of [19, Proposition 2.5], we show that under some conditions, approximate biflatness of $\mathcal{A}$ is provided by the approximate biflatness of $\mathcal{B}$.

\section{AMENABILITY AND PSEUDO- $\phi-$ AMENABILITY}

For the sake of completeness, we first review the basic definitions of abstract Segal algebras; see [4] for more details. Let $\left(\mathcal{A},\|\cdot\|_{\mathcal{A}}\right)$ be a Banach algebra. A Banach algebra $\left(\mathcal{B},\|.\|_{\mathcal{B}}\right)$ is an abstract Segal algebra with respect to $\mathcal{A}$ if $\mathcal{B}$ is a dense left ideal in $\mathcal{A}$ and there exist constants $K>0$ and $M>0$ such that

$$
\|b\|_{\mathcal{A}} \leq K\|b\|_{\mathcal{B}}
$$

and

$$
\|a b\|_{\mathcal{B}} \leq M\|a\|_{\mathcal{A}}\|b\|_{\mathcal{B}}
$$

for all $a \in \mathcal{A}$ and $b \in \mathcal{B}$. Retrieved from the definition of abstract Segal algebras given in [6], we say that $\mathcal{B}$ is an essential abstract Segal algebra with respect to $\mathcal{A}$ if in addition of the previous assumptions, $\mathcal{B}$ is an essential $\mathcal{A}$-module; i.e. $\mathcal{A B}$ is dense in $\left(\mathcal{B},\|\cdot\|_{\mathcal{B}}\right)$, where

$$
\mathcal{A B}=\{a b: a \in \mathcal{A}, b \in \mathcal{B}\} .
$$

We further say that $\left(\mathcal{B},\|.\|_{\mathcal{B}}\right)$ is symmetric if $\mathcal{B}$ is a dense two-sided ideal in $\mathcal{A}$ and the inequality (2.1) holds. Moreover there exists constant $M>0$ such that for all $a \in \mathcal{A}$ and $b \in \mathcal{B}$

$$
\|a b\|_{\mathcal{B}},\|b a\|_{\mathcal{B}} \leq M\|a\|_{\mathcal{A}}\|b\|_{\mathcal{B}}
$$

Throughout the paper, in all the discussions that follow, we use the constants $K$ and $M$ with the meaning ascribed in (2.1), (2.2) and (2.3).

Remark 2.1. It is worth noting that the definition of abstract Segal algebras can be summarized. In fact a Banach algebra $\left(\mathcal{B},\|\cdot\|_{\mathcal{B}}\right)$ is an abstract Segal algebra with respect to the Banach algebra $\left(\mathcal{A},\|\cdot\|_{\mathcal{A}}\right)$, if $\mathcal{B}$ is a dense left ideal in $\mathcal{A}$ and the inequality (2.1) holds. Then one can readily see that (2.2) also is satisfied; see $[2]$.

We commence this section with the following definition. The motivation for this definition arises from [14, Theorem 1.4].

Definition 2.2. Let $\mathcal{A}$ be a Banach algebra and $\phi \in \sigma(\mathcal{A})$. Then $\mathcal{A}$ is called pseudo- $\phi$-amenable if there is a net $\left(a_{\alpha}\right)$ (not necessarily bounded) in $\mathcal{A}$ such that

for all $a \in \mathcal{A}$.

$$
\phi\left(a_{\alpha}\right) \rightarrow 1 \text { and }\left\|a a_{\alpha}-\phi(a) a_{\alpha}\right\|_{\mathcal{A}} \rightarrow 0
$$

Remark 2.3. Note that by replacing $a_{\alpha}$ by $\frac{1}{\phi\left(a_{\alpha}\right)} a_{\alpha}$, one can assume that for each $\alpha, \phi\left(a_{\alpha}\right)=1$, as in [14, Theorem 1.4].

Proposition 2.4. Let $\mathcal{A}$ be a Banach algebra. If $\mathcal{A}$ is pseudo-amenable then $\mathcal{A}$ is pseudo- $\phi$-amenable, for each $\phi \in \sigma(\mathcal{A})$. 
Proof. We use a slightly modified technique used in [7, Proposition 2.5]. Let $\left(m_{\alpha}\right)$ be an approximate diagonal for $\mathcal{A}$. Now for each $\alpha$ write

$$
m_{\alpha}:=\sum_{n=1}^{\infty} a_{n}^{\alpha} \otimes b_{n}^{\alpha},
$$

where $a_{n}^{\alpha}, b_{n}^{\alpha} \in \mathcal{A}$, for each $\alpha$ and $n \geq 1$. Define the linear map $\Phi: \mathcal{A} \widehat{\otimes} \mathcal{A} \rightarrow \mathcal{A}$ by $\Phi(a \otimes b)=a \phi(b)$. Then $\Phi$ is bounded and $\|\Phi\| \leq\|\phi\| \leq 1$. Now set $a_{\alpha}:=\Phi\left(m_{\alpha}\right)$. Then

$$
\phi\left(a_{\alpha}\right)=\sum_{n=1}^{\infty} \phi\left(a_{n}^{\alpha}\right) \phi\left(b_{n}^{\alpha}\right)=\phi\left(\pi_{\mathcal{A}}\left(m_{\alpha}\right)\right) .
$$

Since for each $a \in \mathcal{A},\left\|\pi_{\mathcal{A}}\left(m_{\alpha}\right) a-a\right\|_{\mathcal{A}} \rightarrow 0$, then $\phi\left(a_{\alpha}\right) \rightarrow 1$. Moreover

$$
\begin{aligned}
a a_{\alpha}-\phi(a) a_{\alpha} & =\sum_{n=1}^{\infty} a a_{n}^{\alpha} \phi\left(b_{n}^{\alpha}\right)-\sum_{n=1}^{\infty} a_{n}^{\alpha} \phi\left(b_{n}^{\alpha} a\right) \\
& =\Phi\left(a m_{\alpha}-m_{\alpha} a\right) .
\end{aligned}
$$

It follows that $\left\|a a_{\alpha}-\phi(a) a_{\alpha}\right\|_{\mathcal{A}} \rightarrow 0$ and so the result is proved.

By $\left[1\right.$, Lemma 2.2], $\sigma(\mathcal{B})=\left\{\left.\phi\right|_{\mathcal{B}}: \phi \in \sigma(\mathcal{A})\right\}$, whenever $\mathcal{B}$ is an abstract Segal algebra with respect to $\mathcal{A}$. Now the following result is obtained by the similar arguments given in [1, Proposition 2.3].

Proposition 2.5. Let $\mathcal{A}$ be a Banach algebra and let $\mathcal{B}$ be an abstract Segal algebra with respect to $\mathcal{A}$ and $\phi \in \sigma(\mathcal{A})$. Then $\mathcal{A}$ is pseudo- $\phi$-amenable if and only if $\mathcal{B}$ is pseudo- $\left.\phi\right|_{\mathcal{B}}$-amenable.

Propositions 2.4 and 2.5 yield the following result.

Proposition 2.6. Let $\mathcal{A}$ be a Banach algebra and let $\mathcal{B}$ be an abstract Segal algebra with respect to $\mathcal{A}$. If $\mathcal{B}$ is pseudo-amenable then $\mathcal{A}$ is pseudo- $\phi$-amenable, for each $\phi \in \sigma(\mathcal{A})$.

Remark 2.7. It is known that if $\mathcal{B}$ is amenable then $\mathcal{A}=\mathcal{B}$, as Banach algebras, and so $\mathcal{A}$ is amenable. But in the general case, amenability of $\mathcal{A}$ does not imply amenability of $\mathcal{B}$. For example consider the Lebesgue space $L^{p}(G)$ on locally compact group $G$, for $1 \leq p<\infty$, as defined in [10]. If we take $G$ to be an infinite compact group, then $L^{1}(G)$ is amenable. Whereas for each $1<p<\infty$, $L^{p}(G)$ is a Segal algebra with respect to $L^{1}(G)$, which is not amenable. We refer to [17] for this result and also basic definitions of Segal algebras.

In [19, Corollary 3.2], it is shown that in the class of [SIN]-groups, amenability of $L^{1}(G)$ is equivalent to pseudo-amenability of every Segal algebra. In two next results, we present some assumptions for which amenability of $\mathcal{A}$ implies pseudoamenability of $\mathcal{B}$.

Proposition 2.8. Let $\mathcal{A}$ be a Banach algebra and let $\mathcal{B}$ be an abstract Segal algebra with respect to $\mathcal{A}$. Suppose that $\mathcal{B}$ contains a net $\left(e_{\lambda}\right)$ in its center such that $\left(e_{\lambda}^{2}\right)$ is an approximate identity for $\mathcal{B}$. If $\mathcal{A}$ is amenable then $\mathcal{B}$ is pseudoamenable. 
Proof. Since $\mathcal{A}$ is amenable then it is biflat [5, Theorem 2.9.65] and consequently it is approximately biflat. Now [19, Proposition 2.5] implies that $\mathcal{B}$ is approximately biflat, as well. Since $\mathcal{B}$ has an approximate identity, then [19, Theorem 2.4] follows that $\mathcal{B}$ is pseudo-amenable.

Note that in [19] abstract Segal algebras are always assumed to be essential. However, the proof of [19, Proposition 2.5] is also valid when $\mathcal{B}$ is not necessarily essential.

If $\mathcal{A}$ is amenable then it admits a bounded approximate identity $\left(e_{\lambda}\right)[12$, Proposition 1.16]. If $\mathcal{B}$ contains $\left(e_{\lambda}\right)$, then the following result is obtained.

Corollary 2.9. Let $\mathcal{A}$ be a commutative Banach algebra and let $\mathcal{B}$ be an essential abstract Segal algebra with respect to $\mathcal{A}$. If $\mathcal{A}$ is amenable and $\mathcal{B}$ contains the bounded approximate identity of $\mathcal{A}$, then $\mathcal{B}$ is pseudo-amenable.

Proof. Since $\mathcal{B}$ is essential, it follows that $\left(e_{\lambda}^{2}\right)$ is an approximate identity for $\mathcal{B}$. Now the result is obtained by Proposition 2.8.

The following example shows that the converse of Proposition 2.8 is not necessarily valid.

Example 2.10. For $1 \leq p<\infty$ the Banach sequence algebras $\ell^{p}$, under pointwise multiplication, are pseudo-contractible [7] and so pseudo amenable. Moreover $\ell^{1}$ is an abstract Segal algebra with respect to $\ell^{p}$ and since $\ell^{p}$ does not have an identity, it follows that $\ell^{p}$ is not amenable.

In order to give the desired result for the converse of Proposition 2.8, we shall impose some additional assumptions. First, note that given $u \in \mathcal{B} \widehat{\otimes} \mathcal{B}$, when viewing $u$ as an element of $\mathcal{A} \widehat{\otimes} \mathcal{A}$, more precisely we mean $\iota \otimes \iota(u)$, where $\iota: \mathcal{B} \rightarrow \mathcal{A}$ is the identity embedding. Moreover $\iota \otimes \iota: \mathcal{B} \widehat{\otimes} \mathcal{B} \rightarrow \mathcal{A} \widehat{\otimes} \mathcal{A}$ is clearly a bounded $\mathcal{B}$-bimodule map. If $\mathcal{B}$ is symmetric, then $\iota \otimes \iota$ is bounded $\mathcal{A}$-bimodule.

The next lemma is important and useful for our further arguments. It is obtained immediately.

Lemma 2.11. Let $\mathcal{A}$ be a Banach algebra and let $\mathcal{B}$ be an abstract Segal algebra with respect to $\mathcal{A}$. Then

(i) for each $u \in \mathcal{B} \widehat{\otimes} \mathcal{B}$,

$$
\|\iota \otimes \iota(u)\|_{\mathcal{A} \widehat{\otimes} \mathcal{A}} \leq K^{2}\|u\|_{\mathcal{B} \widehat{\otimes} \mathcal{B}} .
$$

(ii) for each $u \in \mathcal{B} \widehat{\otimes} \mathcal{B}$,

$$
\pi_{\mathcal{A}}(\iota \otimes \iota(u))=\iota\left(\pi_{\mathcal{B}}(u)\right)
$$

Lemma 2.12. Let $\mathcal{A}$ be a Banach algebra and let $\mathcal{B}$ be an abstract Segal algebra with respect to $\mathcal{A}$.

(i) Suppose that $\left(m_{\lambda}\right)$ is a net in $\mathcal{B} \widehat{\otimes} \mathcal{B}$ such that $\left(\iota \otimes \iota\left(m_{\lambda}\right)\right)$ is a bounded net in $\mathcal{A} \widehat{\otimes} \mathcal{A}$ and for each $b \in \mathcal{B},\left\|b m_{\lambda}-m_{\lambda} b\right\|_{\mathcal{B} \widehat{\otimes} \mathcal{B}} \rightarrow 0$. Then

$$
\left\|a \iota \otimes \iota\left(m_{\lambda}\right)-\iota \otimes \iota\left(m_{\lambda}\right) a\right\|_{\mathcal{A} \widehat{\otimes} \mathcal{A}} \rightarrow 0
$$

for each $a \in \mathcal{A}$. 
(ii) Suppose that $m \in \mathcal{B} \widehat{\otimes} \mathcal{B}$ and for each $b \in \mathcal{B}, b m=m b$. Then

$$
a \iota \otimes \iota(m)=\iota \otimes \iota(m) a,
$$

for each $a \in \mathcal{A}$.

(iii) Suppose that $\left(e_{\lambda}\right)$ is an approximate identity for $\mathcal{B}$ that is bounded in $\mathcal{A}$. Then $\left(e_{\lambda}\right)$ is a bounded approximate identity for $\mathcal{A}$.

Proof. We only prove the statement $(i)$. Two other parts can be readily proved. By hypothesis there exists a constant $L>0$ such that for each $\lambda$,

$$
\left\|\iota \otimes \iota\left(m_{\lambda}\right)\right\|_{\mathcal{A} \widehat{\otimes} \mathcal{A}} \leq L .
$$

For $a \in \mathcal{A}$ and $\varepsilon>0$, there is $b \in \mathcal{B}$ and also $\lambda_{0}$ such that

$$
\|a-b\|_{\mathcal{A}}<\frac{\varepsilon}{4 L}
$$

and for each $\lambda \geq \lambda_{0}$

$$
\left\|b m_{\lambda}-m_{\lambda} b\right\|_{\mathcal{B} \widehat{\otimes} \mathcal{B}}<\frac{\varepsilon}{2 K^{2}} .
$$

Using Lemma $2.11(i)$, for each $\lambda \geq \lambda_{0}$ we have

$$
\begin{aligned}
\left\|a \iota \otimes \iota\left(m_{\lambda}\right)-\iota \otimes \iota\left(m_{\lambda}\right) a\right\|_{\mathcal{A} \widehat{\otimes} \mathcal{A}} \leq & \left\|a \iota \otimes \iota\left(m_{\lambda}\right)-b \iota \otimes \iota\left(m_{\lambda}\right)\right\|_{\mathcal{A} \widehat{\otimes} \mathcal{A}} \\
& +\left\|b \iota \otimes \iota\left(m_{\lambda}\right)-\iota \otimes \iota\left(m_{\lambda}\right) b\right\|_{\mathcal{A} \widehat{\otimes} \mathcal{A}} \\
& +\left\|\iota \otimes \iota\left(m_{\lambda}\right) b-\iota \otimes \iota\left(m_{\lambda}\right) a\right\|_{\mathcal{A} \widehat{\otimes} \mathcal{A}} \\
\leq & 2 L\|a-b\|_{\mathcal{A}}+K^{2}\left\|b m_{\lambda}-m_{\lambda} b\right\|_{\mathcal{B} \widehat{\otimes} \mathcal{B}} \\
< & \frac{\varepsilon}{2}+\frac{\varepsilon}{2} \\
= & \varepsilon .
\end{aligned}
$$

It follows that

$$
\left\|a \iota \otimes \iota\left(m_{\lambda}\right)-\iota \otimes \iota\left(m_{\lambda}\right) a\right\|_{\mathcal{A} \widehat{\otimes} \mathcal{A}} \rightarrow 0
$$

which completes the proof.

Proposition 2.13. Let $\mathcal{A}$ be a Banach algebra and let $\mathcal{B}$ be an abstract Segal algebra with respect to $\mathcal{A}$.

(i) Suppose that $\left(m_{\lambda}\right)$ is an approximate diagonal for $\mathcal{B}$ such that $\left(\iota \otimes \iota\left(m_{\lambda}\right)\right)$ is a bounded net in $\mathcal{A} \widehat{\otimes} \mathcal{A}$. Then $\left(\iota \otimes \iota\left(m_{\lambda}\right)\right)$ is a bounded approximate diagonal for $\mathcal{A}$.

(ii) Suppose that $\mathcal{B}$ is pseudo amenable with an approximate diagonal $\left(m_{\lambda}\right)$ such that $\left(\iota \otimes \iota\left(m_{\lambda}\right)\right)$ is bounded in $\mathcal{A} \widehat{\otimes} \mathcal{A}$. Then $\mathcal{A}$ is amenable.

Proof. (i). By Lemma $2.12(i)$,

$$
\left\|a \iota \otimes \iota\left(m_{\lambda}\right)-\iota \otimes \iota\left(m_{\lambda}\right) a\right\|_{\mathcal{A} \widehat{\otimes} \mathcal{A}} \rightarrow 0,
$$

for each $a \in \mathcal{A}$. By hypothesis, $\pi_{\mathcal{B}}\left(m_{\lambda}\right)$ is an approximate identity for $\mathcal{B}$ that is bounded in $\mathcal{A}$. So, by Lemma $2.11(\mathrm{ii})$ and Lemma $2.12(\mathrm{iii}), \pi_{\mathcal{A}}\left(\iota \otimes \iota\left(m_{\lambda}\right)\right)$ is a bounded approximate identity for $\mathcal{A}$. This gives the proposition.

(ii). It is immediately provided by part $(i)$. 
Remark 2.14. Let $\mathcal{A}$ be a Banach algebra and let $\mathcal{B}$ be an abstract Segal algebra with respect to $\mathcal{A}$. Here we investigate previous results about the concept of contractibility and pseudo-contractibility.

(i) $\mathcal{A}$ is contractible if and only if $\mathcal{A}$ is biprojective and unital. It is a known result; see for example [18, section 4, Exercises 4.1.1,4.3.1].

(ii) $\mathcal{B}$ is contractible if and only if $\mathcal{A}$ is contractible and $\mathcal{A}=\mathcal{B}$. This result can be readily verified by the open mapping theorem and part $(i)$.

(iii) Suppose that $\mathcal{B}$ contains a net $\left(e_{\lambda}\right)_{\lambda \in \Lambda}$ in its center such that $\left(e_{\lambda}^{2}\right)_{\lambda \in \Lambda}$ is an approximate identity for $\mathcal{B}$. Then contractibility of $\mathcal{A}$ implies pseudocontractibility of $\mathcal{B}$. Indeed, let $m$ be a diagonal for $\mathcal{A}$ and for each $\lambda \in \Lambda$, let $m_{\lambda}:=e_{\lambda} m e_{\lambda}$. It is readily proved that $\left(m_{\lambda}\right)$ is a central approximate diagonal for $\mathcal{B}$.

(iv) The converse of part (iii) is not in general true. For instance, consider the Banach sequence algebra $\ell^{1}$, discussed in Example 2.10 that is pseudocontractible [7]. Also for $1<p<\infty, \ell^{1}$ is an abstract Segal algebra with respect to $\ell^{p}$, which is not obviously contractible.

(v) Under some additional assumptions, pseudo-contractibility of $\mathcal{A}$ can be provided by pseudo-contractibility of $\mathcal{B}$. For example, if $\left(m_{\lambda}\right)$ is a central approximate diagonal for $\mathcal{B}$ such that $\left(\pi_{\mathcal{A}}\left(\iota \otimes \iota\left(m_{\lambda}\right)\right)\right)$ is a bounded net in $\mathcal{A}$, then $\left(\iota \otimes \iota\left(m_{\lambda}\right)\right)$ is a central approximate diagonal for $\mathcal{A}$. The proof is clearly obtained by Lemma $2.12(\mathrm{ii})$, together with the similar tolls used in Proposition 2.13.

(vi) Let $\mathcal{B}$ be pseudo-contractible with a central approximate diagonal $\left(m_{\lambda}\right)$ such that $\left(\pi_{\mathcal{A}}\left(\iota \otimes \iota\left(m_{\lambda}\right)\right)\right)$ is a bounded net in $\mathcal{A}$. As a consequence of the previous part, one can immediately conclude that $\mathcal{A}$ is pseudo-contractible with a bounded approximate identity.

\section{Approximate Biprojectivity AND Approximate Biflatness}

In order to prove the main results of this section, we require more precise information concerning the tensor product of Banach algebras. It will be given in the following lemma. The proof is straightforward and so will be omitted.

Lemma 3.1. Let $\mathcal{A}$ be a Banach algebra and let $\mathcal{B}$ be a symmetric abstract Segal algebra with respect to $\mathcal{A}$. Then the following assertions hold.

(i) $\mathcal{B} \widehat{\otimes} \mathcal{B}$ is a Banach $\mathcal{A}$-bimodule and for each $a \in \mathcal{A}$ and $u \in \mathcal{B} \widehat{\otimes} \mathcal{B}$,

$$
\|a u\|_{\mathcal{B} \hat{\otimes} \mathcal{B}} \leq M\|a\|_{\mathcal{A}}\|u\|_{\mathcal{B} \widehat{\otimes} \mathcal{B}} \text { and } \quad\|u a\|_{\mathcal{B} \widehat{\otimes} \mathcal{B}} \leq M\|a\|_{\mathcal{A}}\|u\|_{\mathcal{B} \widehat{\otimes} \mathcal{B}}
$$

(ii) If $u \in \mathcal{A} \widehat{\otimes} \mathcal{A}$ and $b, c \in \mathcal{B}$, then buc $\in \mathcal{B} \widehat{\otimes} \mathcal{B}$ and

$$
\|b u c\|_{\mathcal{B} \widehat{\otimes} \mathcal{B}} \leq M^{2}\|b\|_{\mathcal{B}}\|c\|_{\mathcal{B}}\|u\|_{\mathcal{A} \widehat{\otimes} \mathcal{A}} .
$$

Proposition 3.2. Let $\mathcal{A}$ be a Banach algebra and let $\mathcal{B}$ be a symmetric abstract Segal algebra with respect to $\mathcal{A}$. Suppose that $\mathcal{B}$ contains a net $\left(e_{\lambda}\right)_{\lambda \in \Lambda}$ in its center such that $\left(e_{\lambda}^{2}\right)_{\lambda \in \Lambda}$ is an approximate identity for $\mathcal{B}$. If $\mathcal{A}$ is biprojective then $\mathcal{B}$ is approximately biprojective. 
Proof. Since $\mathcal{A}$ is biprojective, there is a bounded $\mathcal{A}$-bimodule morphism $\xi$ : $\mathcal{A} \rightarrow \mathcal{A} \widehat{\otimes} \mathcal{A}$ such that $\pi_{\mathcal{A}} \circ \xi=i d_{\mathcal{A}}$. For each $\lambda \in \Lambda$, define $\xi_{\lambda}: \mathcal{B} \rightarrow \mathcal{B} \widehat{\otimes} \mathcal{B}$ by $\xi_{\lambda}(b):=\xi\left(e_{\lambda} b e_{\lambda}\right)$. Note that $\left(e_{\lambda}\right)_{\lambda \in \Lambda}$ is also in the center of $\mathcal{A}$. It is easy to show that $\xi_{\lambda}$ is a $\mathcal{B}$-bimodule morphism, for each $\lambda \in \Lambda$. We show that $\xi_{\lambda}$ is bounded. Lemma 3.1 together with the fact that $\xi$ is a $\mathcal{A}$-bimodule morphism yield that

$$
\begin{aligned}
\left\|\xi_{\lambda}(b)\right\|_{\mathcal{B} \hat{\otimes} \mathcal{B}} & =\left\|\xi\left(e_{\lambda} b e_{\lambda}\right)\right\|_{\mathcal{B} \hat{\otimes} \mathcal{B}} \\
& =\left\|e_{\lambda} \xi(b) e_{\lambda}\right\|_{\mathcal{B} \widehat{\otimes} \mathcal{B}} \\
& \leq M^{2}\|\xi(b)\|_{\mathcal{A} \widehat{\otimes} \mathcal{A}}\left\|e_{\lambda}\right\|_{\mathcal{B}}^{2} \\
& \leq M^{2}\|\xi\|\|b\|_{\mathcal{A}}\left\|e_{\lambda}\right\|_{\mathcal{B}}^{2} \\
& \leq M^{2} K\|\xi\|\|b\|_{\mathcal{B}}\left\|e_{\lambda}\right\|_{\mathcal{B}}^{2} .
\end{aligned}
$$

It follows that $\xi_{\lambda}$ is bounded and

$$
\left\|\xi_{\lambda}\right\| \leq M^{2} K\|\xi\|\left\|e_{\lambda}\right\|_{\mathcal{B}}^{2}
$$

Moreover for each $b \in \mathcal{B}$

$$
\pi_{\mathcal{B}} \circ \xi_{\lambda}(b)=\pi_{\mathcal{B}}\left(\xi\left(e_{\lambda} b e_{\lambda}\right)\right)=\pi_{\mathcal{A}}\left(\xi\left(e_{\lambda} b e_{\lambda}\right)\right)=e_{\lambda} b e_{\lambda}=b e_{\lambda}^{2} .
$$

Since $\left(e_{\lambda}^{2}\right)_{\lambda \in \Lambda}$ is an approximate identity for $\mathcal{B}$, then

$$
\left\|\pi_{\mathcal{B}} \circ \xi_{\lambda}(b)-b\right\|_{\mathcal{B}} \rightarrow 0 .
$$

It follows that $\left(\xi_{\lambda}\right)_{\lambda \in \Lambda}$ is a net of bounded $\mathcal{B}$-bimodule morphisms and therefore $\mathcal{B}$ is approximately biprojective.

Proposition 3.3. Let $\mathcal{A}$ be a Banach algebra and let $\mathcal{B}$ be an abstract Segal algebra with respect to $\mathcal{A}$. Suppose that $\mathcal{B}$ contains a net $\left(e_{\lambda}\right)_{\lambda \in \Lambda}$ in its center such that $\left(e_{\lambda}^{2}\right)_{\lambda \in \Lambda}$ is an approximate identity for $\mathcal{A}$. If $\mathcal{B}$ is biprojective then $\mathcal{A}$ is approximately biprojective.

Proof. Since $\mathcal{B}$ is biprojective, there is a bounded $\mathcal{B}$-bimodule morphism $\xi: \mathcal{B} \rightarrow$ $\mathcal{B} \widehat{\otimes} \mathcal{B}$ such that $\pi_{\mathcal{B}} \circ \xi=i d_{\mathcal{B}}$. For each $\lambda \in \Lambda$, define $\xi_{\lambda}: \mathcal{A} \rightarrow \mathcal{A} \widehat{\otimes} \mathcal{A}$ by

$$
\xi_{\lambda}(a):=\iota \otimes \iota\left(\xi\left(e_{\lambda} a e_{\lambda}\right)\right) .
$$

We show that for each $\lambda \in \Lambda, \xi_{\lambda}$ is a bounded $\mathcal{A}$-bimodule morphism. Take $a, c \in \mathcal{A}$. Note that $\left(e_{\lambda}\right)_{\lambda \in \Lambda}$ is also in the center of $\mathcal{A}$. Since $\xi$ is a $\mathcal{B}$-bimodule morphism and $e_{\lambda} c \in \mathcal{B}$, then

$$
\begin{aligned}
\xi_{\lambda}(c a) & =\iota \otimes \iota\left(\xi\left(e_{\lambda} c a e_{\lambda}\right)\right)=e_{\lambda} c \iota \otimes \iota\left(\xi\left(a e_{\lambda}\right)\right) \\
& =c e_{\lambda} \iota \otimes \iota\left(\xi\left(a e_{\lambda}\right)\right)=c \iota \otimes \iota\left(\xi\left(e_{\lambda} a e_{\lambda}\right)\right) \\
& =c \xi_{\lambda}(a) .
\end{aligned}
$$

Similarly $\xi_{\lambda}(c a)=\xi_{\lambda}(c) a$. Also by Lemma $2.11(i)$ we have

$$
\begin{aligned}
\left\|\xi_{\lambda}(a)\right\|_{\mathcal{A} \widehat{\otimes} \mathcal{A}} & =\left\|\iota \otimes \iota\left(\xi\left(e_{\lambda} a e_{\lambda}\right)\right)\right\|_{\mathcal{A} \widehat{\otimes} \mathcal{A}} \\
& \leq K^{2}\left\|\xi\left(e_{\lambda} a e_{\lambda}\right)\right\|_{\mathcal{B} \widehat{\otimes} \mathcal{B}} \\
& \leq K^{2}\|\xi\|\left\|e_{\lambda} a e_{\lambda}\right\|_{\mathcal{B}} \\
& \leq K^{2} M\|\xi\|\left\|e_{\lambda}\right\|_{\mathcal{B}}^{2}\|a\|_{\mathcal{A}}
\end{aligned}
$$


and consequently

$$
\left\|\xi_{\lambda}\right\| \leq K^{2} M\|\xi\|\left\|e_{\lambda}\right\|_{\mathcal{B}}^{2}
$$

Moreover

$$
\begin{aligned}
\pi_{\mathcal{A}} \circ \xi_{\lambda}(a) & =\pi_{\mathcal{A}}\left(\iota \otimes \iota\left(\xi\left(e_{\lambda} a e_{\lambda}\right)\right)\right) \\
& =\iota\left(\pi_{\mathcal{B}} \circ \xi\left(e_{\lambda} a e_{\lambda}\right)\right) \\
& =e_{\lambda} a e_{\lambda} .
\end{aligned}
$$

Since $\left(e_{\lambda}^{2}\right)_{\lambda \in \Lambda}$ is an approximate identity for $\mathcal{A}$, then

$$
\left\|\pi_{\mathcal{A}} \circ \xi_{\lambda}(a)-a\right\|_{\mathcal{A}} \rightarrow 0 .
$$

It follows that $\left(\xi_{\lambda}\right)_{\lambda \in \Lambda}$ is a net of bounded $\mathcal{A}$-bimodule morphisms. Hence, $\mathcal{A}$ is approximately biprojective.

By [19, Proposition 2.5], if $\mathcal{B}$ contains a net $\left(e_{\lambda}\right)_{\lambda \in \Lambda}$ in its center such that $\left(e_{\lambda}^{2}\right)_{\lambda \in \Lambda}$ is an approximate identity for $\mathcal{B}$, then $\mathcal{B}$ is approximately biflat, whenever so is $\mathcal{A}$. As the main result in the present paper, we prove the converse of this result, under the assumption that $\left(e_{\lambda}\right)_{\lambda \in \Lambda}$ is an approximate identity for $\mathcal{A}$. It requires an elementary lemma.

Lemma 3.4. Let $\mathcal{A}$ be a Banach algebra and let $\mathcal{B}$ be an abstract Segal algebra with respect to $\mathcal{A}$. Then the following assertions hold.

(i) If $f \in \mathcal{A}^{*}$ and $f^{\sharp}=\left.f\right|_{\mathcal{B}}$, the restricted function of $f$ on $\mathcal{B}$, then $f^{\sharp} \in \mathcal{B}^{*}$ and $\left\|f^{\sharp}\right\|_{\mathcal{B}^{*}} \leq K\|f\|_{\mathcal{A}^{*}}$.

(ii) If $f \in \mathcal{B}^{*}$ and $b \in \mathcal{B}$, then b. $f \in \mathcal{A}^{*}$ and

$$
\|b . f\|_{\mathcal{A}^{*}} \leq M\|b\|_{\mathcal{B}}\|f\|_{\mathcal{B}^{*}}
$$

where $(b . f)(a):=f(a b)$ for each $a \in \mathcal{A}$. If $\mathcal{B}$ is symmetric, then $f . b \in \mathcal{A}^{*}$ and

$$
\|f . b\|_{\mathcal{A}^{*}} \leq M\|b\|_{\mathcal{B}}\|f\|_{\mathcal{B}^{*}},
$$

where $(f . b)(a):=f(b a)$ for each $a \in \mathcal{A}$.

(iii) For $f \in(\mathcal{A} \widehat{\otimes} \mathcal{A})^{*}$, define the linear map $f^{\sharp}: \mathcal{B} \widehat{\otimes} \mathcal{B} \rightarrow \mathbb{C}$ by

$$
f^{\sharp}(b \otimes c):=f(\iota \otimes \iota(b \otimes c)),
$$

for all $b, c \in \mathcal{B}$. Then $f^{\sharp} \in(\mathcal{B} \widehat{\otimes} \mathcal{B})^{*}$ and

$$
\left\|f^{\sharp}\right\|_{(\mathcal{B} \widehat{\otimes} \mathcal{B})^{*}} \leq K^{2}\|f\|_{(\mathcal{A} \widehat{\otimes} \mathcal{A})^{*}} .
$$

Proof. The parts $(i)$ and $(i i)$ follow immediately. For the last part note that $f^{\sharp}=(\iota \otimes \iota)^{*}(f)$ and also

$$
\left\|(\iota \otimes \iota)^{*}\right\|=\|\iota \otimes \iota\| \leq K^{2} .
$$

Thus the result is obtained.

Theorem 3.5. Let $\mathcal{A}$ be a Banach algebra and let $\mathcal{B}$ be a symmetric abstract Segal algebra with respect to $\mathcal{A}$. Suppose that $\mathcal{B}$ contains a net $\left(e_{\lambda}\right)_{\lambda \in \Lambda}$ in its center such that it is an approximate identity for $\mathcal{A}$. If $\mathcal{B}$ is approximately biflat then so is $\mathcal{A}$. 
Proof. By hypothesis, there is a net

$$
\theta_{\delta}:(\mathcal{B} \widehat{\otimes} \mathcal{B})^{*} \rightarrow \mathcal{B}^{*}
$$

$(\delta \in \Delta)$ of bounded $\mathcal{B}$-bimodule morphisms such that

$$
W^{*} O T-\lim _{\delta} \theta_{\delta} \circ \pi_{\mathcal{B}}{ }^{*}=i d_{\mathcal{B}^{*}}
$$

For each $\lambda \in \Lambda$, define the linear map $T_{\lambda}: \mathcal{B}^{*} \rightarrow \mathcal{A}^{*}$ by

$$
T_{\lambda}(f):=f . e_{\lambda} \quad\left(f \in \mathcal{B}^{*}\right) .
$$

Also let $T:(\mathcal{A} \widehat{\otimes} \mathcal{A})^{*} \rightarrow(\mathcal{B} \widehat{\otimes} \mathcal{B})^{*}$ defined by $f \mapsto f^{\sharp}$ that is clearly well defined by Lemma 3.4. Let $E=\Lambda \times \Delta^{\Lambda}$ be directed by the product ordering, and for each $\beta=\left(\lambda,\left(\delta_{\lambda^{\prime}}\right)_{\lambda^{\prime} \in \Lambda}\right)$ belonging to $E$ define

$$
\theta_{\beta}:(\mathcal{A} \widehat{\otimes} \mathcal{A})^{*} \rightarrow \mathcal{A}^{*}
$$

by $\theta_{\beta}:=T_{\lambda} \circ \theta_{\delta_{\lambda}} \circ T$. Since $\left(e_{\lambda}\right)$ is in the center of $\mathcal{B}$ it is also in the center of $\mathcal{A}$, and therefore $T_{\lambda}$ is a bounded $\mathcal{A}$-bimodule map. Also, $\theta_{\delta_{\lambda}}$ is a bounded $\mathcal{B}$-bimodule map so, by density of $\mathcal{B}$ in $\mathcal{A}, \theta_{\delta_{\lambda}}$ is also a bounded $\mathcal{A}$-bimodule map. Moreover,

$$
\iota \otimes \iota: \mathcal{B} \widehat{\otimes} \mathcal{B} \rightarrow \mathcal{A} \widehat{\otimes} \mathcal{A}
$$

is clearly a bounded $\mathcal{A}$-bimodule map, so $T=(\iota \otimes \iota)^{*}$ is as well. Hence, $\theta_{\beta}=$ $T_{\lambda} \circ \theta_{\delta_{\lambda}} \circ T$ is a bounded $\mathcal{A}$-bimodule map. Finally we show that

$$
W^{*} O T \lim _{\beta} \theta_{\beta} \circ \pi_{\mathcal{A}}^{*}=i d_{\mathcal{A}^{*}} .
$$

According to the explanations given in Introduction, it suffices to show that for each $g \in \mathcal{A}^{*}$ and $a \in \mathcal{A}$

$$
\lim _{\beta} \rho_{g, a}\left(\theta_{\beta} \circ \pi_{\mathcal{A}}^{*}\right)=\rho_{g, a}\left(i d_{\mathcal{A}^{*}}\right)
$$

By Lemma $2.11(i i),\left(g \circ \pi_{\mathcal{A}}\right)^{\sharp}=g^{\sharp} \circ \pi_{\mathcal{B}}$. Thus

$$
\begin{aligned}
\lim _{\beta} \rho_{g, a}\left(\theta_{\beta} \circ \pi_{\mathcal{A}}^{*}\right) & =\lim _{\beta}\left\langle\theta_{\beta} \circ \pi_{\mathcal{A}}^{*}(g), a\right\rangle \\
& =\lim _{\lambda} \lim _{\delta}\left\langle\left(T_{\lambda} \circ \theta_{\delta} \circ T\right)\left(g \circ \pi_{\mathcal{A}}\right), a\right\rangle \\
& =\lim _{\lambda} \lim _{\delta}\left\langle\left(\theta_{\delta} \circ T\right)\left(g \circ \pi_{\mathcal{A}}\right), e_{\lambda} a\right\rangle \\
& =\lim _{\lambda} \lim _{\delta}\left\langle\theta_{\delta}\left(\left(g \circ \pi_{\mathcal{A}}\right)^{\sharp}\right), e_{\lambda} a\right\rangle \\
& =\lim _{\lambda} \lim _{\delta}\left\langle\theta_{\delta}\left(g^{\sharp} \circ \pi_{\mathcal{B}}\right), e_{\lambda} a\right\rangle \\
& =\lim _{\lambda}\left\langle g^{\sharp}, e_{\lambda} a\right\rangle \\
& =\lim _{\lambda}\left\langle g, e_{\lambda} a\right\rangle \\
& =\langle g, a\rangle \\
& =\left\langle i d_{\mathcal{A}^{*}}(g), a\right\rangle,
\end{aligned}
$$

that in which, we have used the iterated limit theorem from of [16, page 69]. It completes the proof. 
Acknowledgements. The author is very grateful to the anonymous referee for his/her constructive comments and suggestions on the manuscript and very careful reading of the paper. In particular, his constructive and invaluable suggestions led to the improvement of the presentation of the paper. This research was partially supported by the Banach algebra Center of Excellence for Mathematics, University of Isfahan.

\section{REFERENCES}

1. M. Alaghmandan, R. Nasr Isfahani and M. Nemati, Character amenability and contractibility of abstract Segal algebras, Bull. Aust. Math. Soc. 82 (2010), no. 2, 274-281.

2. B.A. Barnes, Banach algebras which are ideals in a Banach algebra, Pacific J. Math. 38 (1971), no. 1, 1-7.

3. A. Bodaghi, M. Eshaghi Gordji and A.R. Medghalchi, A generalization of the weak amenability of Banach algebras, Banach J. Math. Anal. 3 (2009), no. 1, 131-142.

4. J.T. Burnham, Closed ideals in subalgebras of Banach algebras. I, Proc. Amer. Math. Soc. 32 (1972), 551-555.

5. H.G. Dales, Banach algebras and automatic continuity, Oxford University Press, 2000.

6. B.E. Forrest, N. Spronk and P.J. Wood, Operator Segal algebras in Fourier algebras, Studia Math. 179 (2007), no. 3, 277-295.

7. F. Ghahramani and Y. Zhang, Pseudo-amenable and pseudo-contractible Banach algebras, Math. Proc. Cambridge Philos. Soc. 142 (2007), no. 1, 111-123.

8. A.Ya. Helemskii, Banach and Locally Convex Algebras, The Clarendon Press Oxford University Press, New York, 1993.

9. A.Ya. Helemskii, The homology of Banach and topological algebras. Translated from the Russian by Alan West, Mathematics and its Applications (Soviet Series), 41, Kluwer Academic Publishers Group, Dordrecht, 1989, xx+334 pp.

10. E. Hewitt and K.A. Ross, Abstract harmonic analysis. I, Second Edition, 115, SpringerVerlag, Berlin, 1979.

11. Z. Hu, M.S. Monfared and T. Traynor, On character amenable Banach algebras, Studia Math. 193 (2009), no. 1, 53-78.

12. B.E. Johnson, Cohomology in Banach Algebras, Mem. Amer. Math. Soc. 127, Providence, R.I., 1972.

13. B.E. Johnson, Approximate diagonals and cohomology of certain annihilator Banach algebras, Amer. J. Math. 94 (1972), 685-698.

14. E. Kaniuth, A.T. Lau and J. Pym, On $\phi$-amenability of Banach algebras, Math. Proc. Cambridge Philos. Soc. 144 (2008), no. 1, 85-96.

15. E. Kaniuth, A.T. Lau and J. Pym, On character amenability of Banach algebras, J. Math. Anal. Appl. 344 (2008), no. 2, 942-955.

16. J.L. Kelley, General topology, Reprint of the 1955 edition [Van Nostrand, Toronto, Ont.], Graduate Texts in Mathematics, no. 27, Springer-Verlag, New York, 1975.

17. H. Reiter, $L^{1}$-algebras and segal algebras, Lecture Notes in Mathematics, 231, SpringerVerlag, Berlin, 1971.

18. V. Runde, Lectures on amenability, Lecture Notes in Mathematics, 1774, Springer-Verlag, Berlin, 2002.

19. E. Samei, N. Spronk and R. Stokke, Biflatness and pseudo-amenability of Segal algebras, Canad. J. Math. 62 (2010), no. 4, 845-869.

20. Y. Zhang, Nilpotent ideals in a class of Banach algebras, Proc. Amer. Math. Soc. 127 (1999), no. 11, 3237-3242.

Department of Mathematics, University of Isfahan, P. O. Box 81746-73441, ISFAHAN, IRAN.

E-mail address: f.abtahi@sci.ui.ac.ir; abtahif2002@yahoo.com 